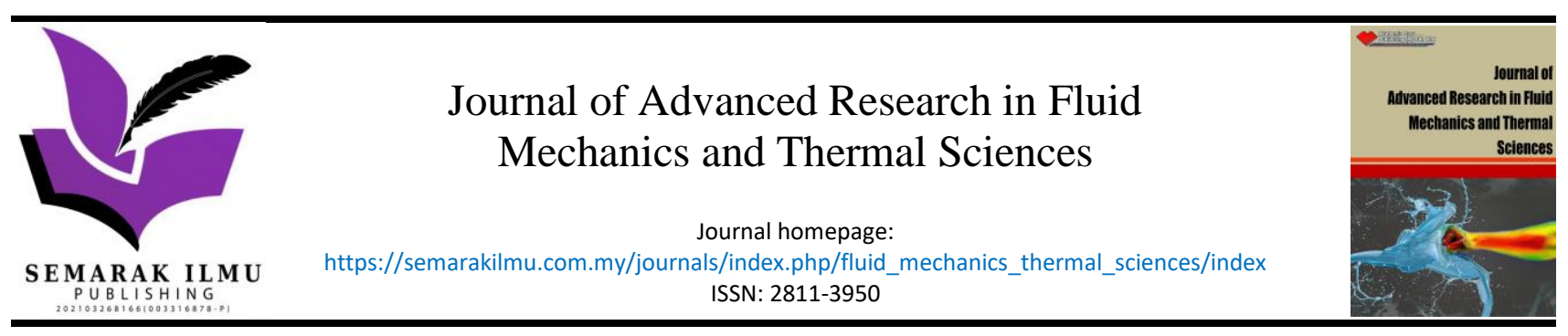

\title{
Advanced Pneumatic Mechanical System for Water Treatment Application: Dosing Rate Assessment for Lime Dosage
}

\author{
Farid Othman ${ }^{1,2}$, Nur Azam Abdullah ${ }^{1,2,{ }^{*}}$, Erwin Sulaeman ${ }^{1}$, Sher Afghan Khan ${ }^{1}$ \\ 1 Department of Mechanical and Aerospace Engineering, Kulliyyah of Engineering, International Islamic University Malaysia, Jalan Gombak, \\ 53100, Kuala Lumpur, Malaysia \\ 2 Aqua Oleo Sdn Bhd, B-6-2A, Jalan Prima Saujana 2G. Taman Prima Saujana, 43000, Kajang, Selangor Darul Ehsan, Malaysia
}

\section{$\begin{array}{ll}\text { ARTICLE INFO } & \text { ABSTRACT }\end{array}$}

\section{Article history:}

Received 14 August 2021

Received in revised form 10 December 2021

Accepted 23 December 2021

Available online 18 January 2022

\begin{abstract}
This paper presents a new developed pneumatic mechanism for lime dosing system in water treatment application. Technically, a choke formation due to scaling problem in such existing water treatment system utilising pump as the transferring medium has caused the high cost of operation due to scheduling maintenance and replacement of pump tubing. In that sense, the pneumatic system will be a new advancement in replacing the transferring medium. In this work, the air regulator is used to control air pressure at several levels. At the same time, there is one valve that is installed to control the airflow the regulator (air compressor) to the pneumatic cylinder cartridge. The assessment is conducted for water with $10 \%$ of lime concentration on water sample 25 PPM as the standard lime concentration for water treatment plant as the domestic usage in Malaysia. The trend of dosing rate for the present novel lime dosage utilising a pneumatic system shows consistency of increment with the increment of applied air pressure during the dosing process.
\end{abstract}

\section{Introduction}

Water is one of the most crucial elements for living creatures. Based on Kumar and Puri [1], Over and above one billion people worldwide do not have instant access to a sufficient and reliable water accumulation surpassing 800 million of those unsaved live in rural regions. On more serious matters, drinking water contamination situations can conceive interruptions in treated water supply operation, which could significantly impact public health and resolve community concerns in drinking water quality [2].

One of the common use substances uses in the water treatment plant is lime [3]. Some reasons for the utilisation of lime in the water treatment system since lime works a significant role in flotation by regulating pulp chemistry and collector adsorption, including as the most economical and regularly employed reagent for $\mathrm{pH}$ control in flotation [4]. In such advanced development in nanotechnology, Wang et al., [5] conducted comparative research on the removal of $\mathrm{Pb}$ (II) and $\mathrm{Zn}$ (II) by using lime

\footnotetext{
* Corresponding author.

E-mail address: azam@iium.edu.my

https://doi.org/10.37934/arfmts.91.1.4655
} 
and nanoscale zero-valent iron (nZVI). This investigation was carried out to examine the effectiveness of nanotechnology for water treatment is whether there are benefits of these nanomaterials over established water treatment reagents. In another hand, a study on the flow pattern of air-water has been performed experimentally to inspect five unique flow patterns: plug, slug-annular, churn, bubbly, and annular, and on a transparent mini pipe with slope of $45^{\circ}$ to the horizontal plane $[6,7]$.

For instance, most existing water treatment plants currently operate the pump system as their core to transmit the treated water for domestic usage. In another hand, Xu et al., [8] has designed and applied such an automatic lime dosing system in metal pickling for wastewater treatment. He stated that the conventional lime dosing system could trigger some severe dust contamination, inadequate working conditions, high labour strength required, low dosing precision, non-uniform solution concentration, and still limited in term of automatic control.

However, a common problem in the pump system for dosing lime substance is the clog due to scaling formation. The sediment produced by the mixture between water and lime made the scaling process grow and consequently result in scale production since lime is considered as a soil organic (sandy) material. In a severe case, the clog may cause a burst on the pump since more power is required to produce higher pressure to transmit the mixing water to be treated. This situation has triggered the water flow to be ineffective and reduce the pump performance [9]. From that perspective, the conventional pump system may prompt higher operational cost due to its frequent maintenance requirement. Some other research has evaluated the inherent effect of extreme weather on water quality and disinfection by the scale generation adopting the laboratory simulation [10]. In a different viewpoint, such discrepancies exist between the convective heat transfer for fluid flow within macro and micro pipes [11].

Another crucial element in assessing the water treatment plant is the Particle per million (PPM), where it is the measurement of an alienate substance with the mixture of water in the water tank. For example, this issue in been infiltrated by Mintenig et al., [12], where the identification of microplastics $(>20 \mu \mathrm{m})$ was assessed by the implementation of Fourier Transform Infrared Spectroscopy (FTIR) imaging for the water purification process. In severe case, the contaminated water supply would consist of high toxic fluoride, and would be a higher PPM level [13]. One study was carried out by Nariyan et al., [14] using an unconventional approach such that iodides and bromides at parts per million concentrations since those chemicals respond with natural organic matters following the oxidative disinfection process and produce toxic disinfectant by-products. They found that these toxic substances could be eliminated by using a novel bismuth composite material.

For developing such great transmission of fluid, technically an advanced approach has been employed by the embedded pneumatic system in the plant. Regarding the effectiveness of power dissipation Chen et al., [15] embedded airpower meter (APM) to measure the energy consumption of flow in pneumatic cylinder actuator system. The cylinder system is developed based on four comparisons: state equation of air, energy equation, motion equation and flow equation. The prototype computes the pressure fluctuation in the charge and releases side of the cylinder and the piston's displacement and velocity. For some improvements, Shi et al., [16] has developed a program to estimate the power of the pneumatic system and explore their potential in several applications. They suggested a necessity to improve the performance of pneumatic systems and the implied components related to compressed air power.

One way of assessing the quality of water flow is via the measurement of the chemical dosing rate. For example, Bonton et al., [17] has conveyed a comparative life cycle evaluation of two water treatment plants, an enhanced standard plant and another nanofiltration plant. Based on their objective, the study showed very different results for the two plants, attracting consideration to the value of the choice of water treatment chemicals and energy sources. This study intends to 
investigate the dosing rate of lime dosage at specific chemical concentrations using a pneumatic system. To the authors' knowledge, this is the first time a lime dosing rate measurement is through in a pneumatic system assessed to the in-water treatment.

\section{Methodology}

\subsection{Flow Rate Measurement}

In this investigation, the flow rate computation is essential in learning the desired lime dosing rate, which would be essential in designing the pneumatic system. In that case, the calculation on pressure changes is required to regulate the flow rate as the pneumatic system is assembled between several segments, such as air compressor, $2 x$ column cylinders and indicator needle valve. In this design, the control needle valve is utilised to regulate airflow.

The flow rate, $\mathrm{Q}$ is measured as the inlet airflow speed, $v_{1}$ at the inlet, opening throat area multiplied with $A_{1}$ which is equivalent as the outlet water flow $v_{2}$ multiplied with the outlet throat area, $A_{2}$ as shown in Eq. (1).

$Q=v_{1} A_{1}=v_{2} A_{2}$

As mentioned earlier that the control needle valve is utilised in controlling the airflow, the inlet and outlet speed would be translated to the changes of pressure as in Eq. (2).

$P_{1}-P_{2}=\left(v_{2}^{2}-v_{1}^{2}\right)$

Later, Eq. (1) and Eq. (2) are combined in the sense of the relationship with these parameters as in Eq. (3).

$\mathrm{Q}=A_{1} \sqrt{\frac{2}{\rho} \frac{P_{1}-P_{2}}{\left(\frac{A_{1}}{A_{2}}\right)^{2}-1}}=A_{2} \sqrt{\frac{2}{\rho} \frac{P_{1}-P_{2}}{\left(\frac{A_{2}}{A_{1}}\right)^{2}-1}}$

From the flow rate, $\mathrm{Q}$ can be plotted on graph flow rate versus time in this research experiment to know the dosing per time and the effectiveness of this pneumatic lime dosing system by using the pneumatic method compared to the metering pump system.

\subsection{Dosing Rate Measurement}

In addition, the dosing calculation also one of the important elements to be considered since the particle of chemical would be relying on this relationship. The dosing rate is measured as in (4), where the particle per minute, PPM depends on the rate of induced particle and the concentration of the mixture between lime and water, $C$. For instance, the pneumatic cylinder airflow that can be related to the flow rate, $Q$ as in Eq. (4).

$D=\frac{Q \times P P M \times 100}{1000 \times C}$

To summarise, several components that are essential in assessing the dosing rate measurement is presented in Table 1. 


\section{Table 1}

Summary on components and control parameters utilised in this study

\begin{tabular}{|c|c|c|}
\hline Type of cylinder & Components & Control Parameters \\
\hline $\begin{array}{l}\text { Double-acting } \\
\text { cylinders }\end{array}$ & $\begin{array}{ll}\text { - } & \text { Piston Area (Bore } \\
& \text { x3.1415) } \\
\text { - } \quad \text { Road Area (for double- } \\
\text { acting cylinders) }\end{array}$ & $\begin{array}{l}\text { - } \quad \text { Pressure valve } \\
\text { - } \quad \text { Cycles per minutes }\end{array}$ \\
\hline
\end{tabular}

For a single-acting cylinder, the air consumption that is used in inducing the pressure as in Cubic feet per minute is as in Eq. (5), where $A$ is the piston area, $S$ is the applied stroke, and $C$ is the cycles per minute.

$C F M=\frac{A \times S \times C}{1728}$

Considering this research utilises double-acting of air consumption; consequently, the proportion of the parameters should be adjusted. In specific employment, the double-acting cylinder may be presumed to prolong and retract at a different rate. In that matter, the condition changes the deliberation of air consumption. In this situation, the air consumption of each stroke could be counted separately as in Eq. (6), where EC is the extended cycles per minute (ignoring dwell time) and $\mathrm{RC}$ is the retract cycles per minute (disregarding dwell time).

$C F M=\frac{A \times S \times E C}{1728}+[(A-R) \times S \times R C]$

The pneumatic system will utilise an air compression mechanism in order to execute the kinematic motion of two designed calibration columns unit. The expected results will comprise the dosing rate compression efficiency throughout the mechanical system. The schematic diagram is demonstrated in Figure 1.

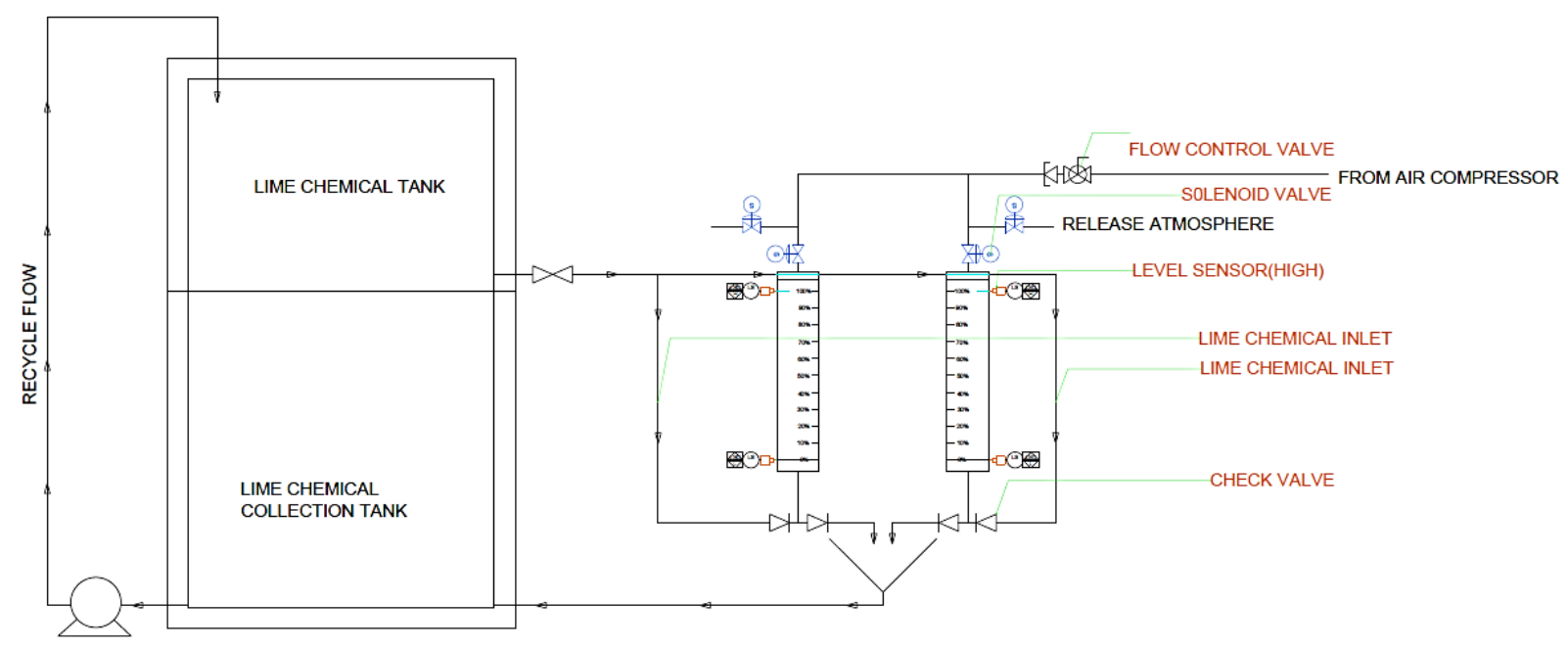

Fig. 1. Diagram of the design pneumatic system fluid flow transfer

\subsection{Prototype Development}

Figure 2 presents the fabricated ideal of the designed water treatment plant employing an upperlevel pneumatic system. This arrangement comprises devices that play a vital function in carrying the dosing in the preparation tank to the dosing point. It works adversely as a pump but solely used by 
pneumatic air as energy relocation. Therefore, this system's critical equipment such as the pneumatic column, solenoid valve, and air regulator controls the airflow as an energy force to carry the dosage. Moreover, this system uses a rotameter calibration flow for calibrating dosing. From the data rotameter calibration, it can gather the total dosing flow that carrier to the dosing point. A ready functioning pneumatic system for the water treatment plant is shown in Figure 3.

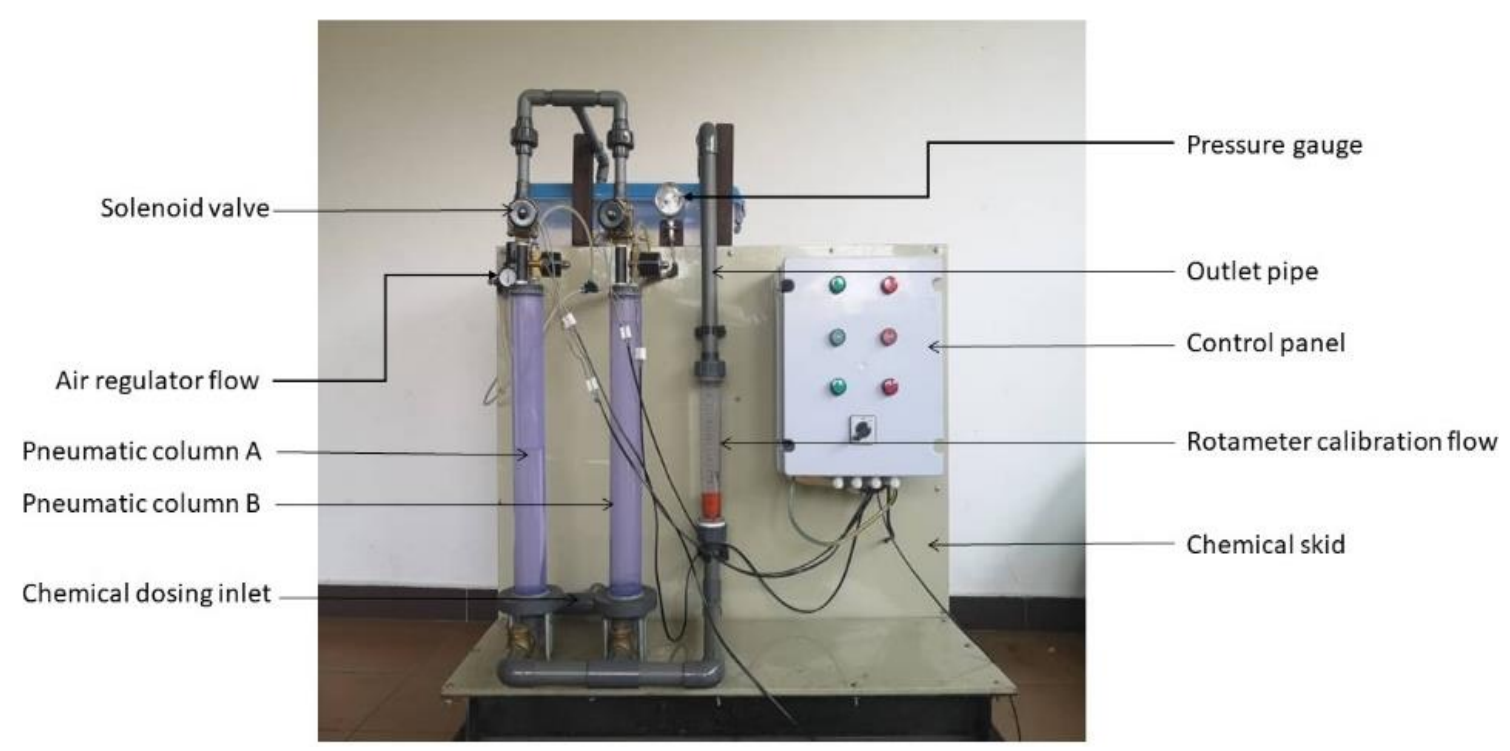

Fig. 2. Developed pneumatic system prototype for the water treatment plant

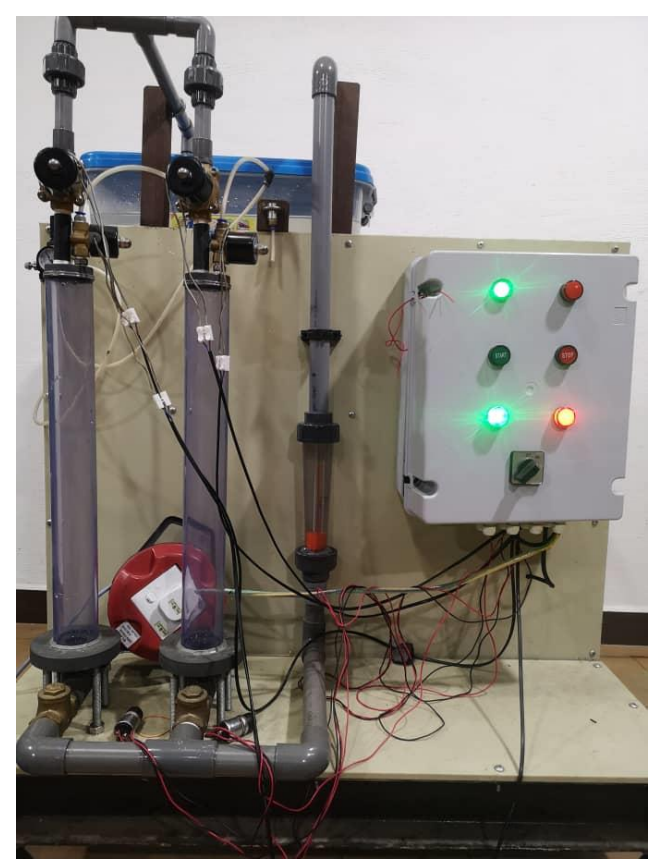

Fig. 3. Fully function pneumatic system for the water treatment plant 


\section{Results}

\subsection{Dosing Rate Assessment}

This section discusses the results obtained from the air pressure variations through valve opening on a flowing $10 \%$ of lime dosing concentration, with 25 Particle per million (PPM) of the sampling examined water. Figure 4 shows the indicator of air pressure released at the measured instant, and Figure 5 demonstrated the sample of dosing rate measurement at $0.3 \mathrm{MPa}$ for $40 \%$ valve opening.

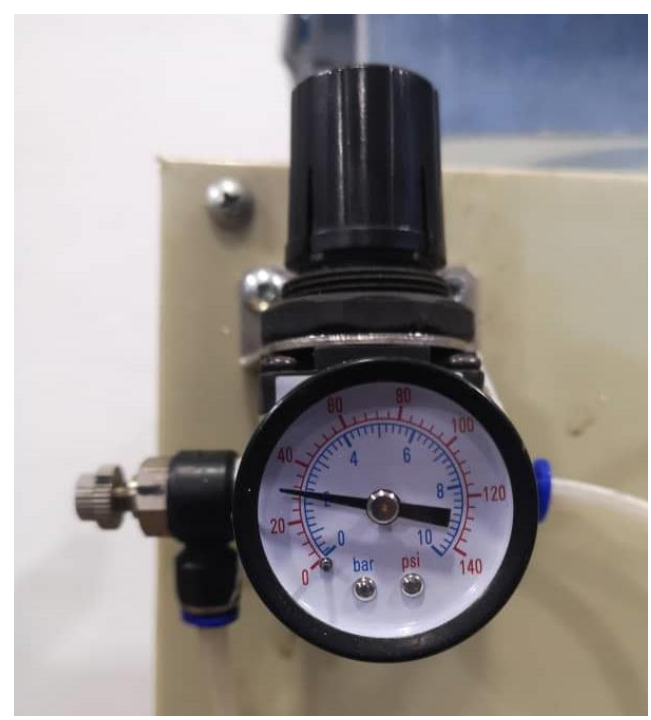

Fig. 4. Sample of air pressure measurement at $\mathrm{P}=0.2 \mathrm{MPa}$

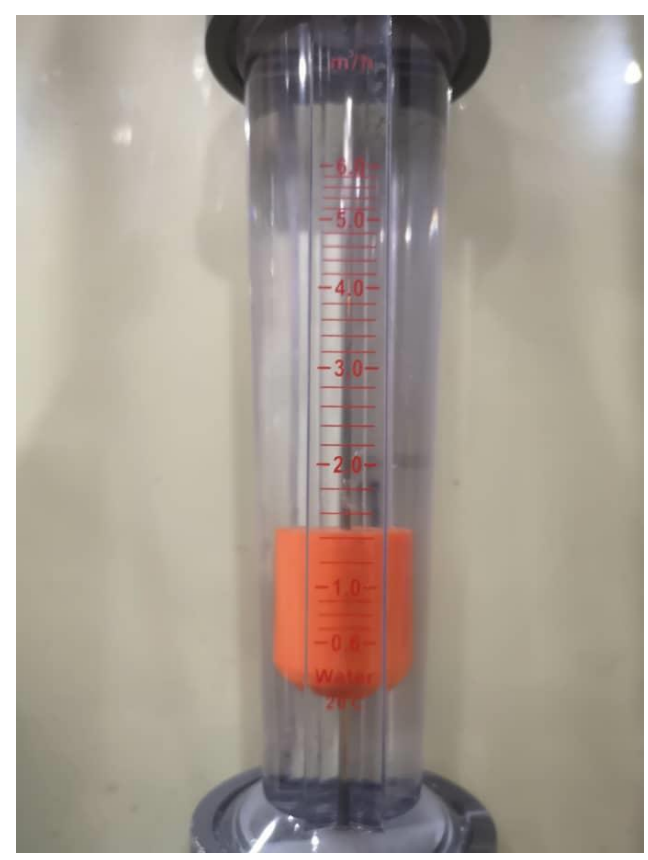

Fig. 5. Sample of dosing rate measurement at $\mathrm{P}=0.3 \mathrm{MPa}$ for $40 \%$ valve opening 
Figure 6 presents the rate of lime dosing throughout the present pneumatic system for air pressure variation released into the pneumatic column. All of the plotted graphs show that the lime dosing rate is increased exponentially with respect to the air released pressure.

The trend of having different released air pressure could be observed in Figure 6, where the dosing rate is increased at higher valve opening for several air pressure ranges. As the sample water is for 25 PPM and $10 \%$ lime concentration on that view, the amount of required flow rate, $Q$, would be obtained through Eq. (4).

Table 2 presents the airflow rate, $Q$ at different valve opening for the sample of 25 PPM, and the relation is demonstrated in Figure 7 . It is observed that the airflow rate is proportional to the lime dosing rate with respect to pressure.

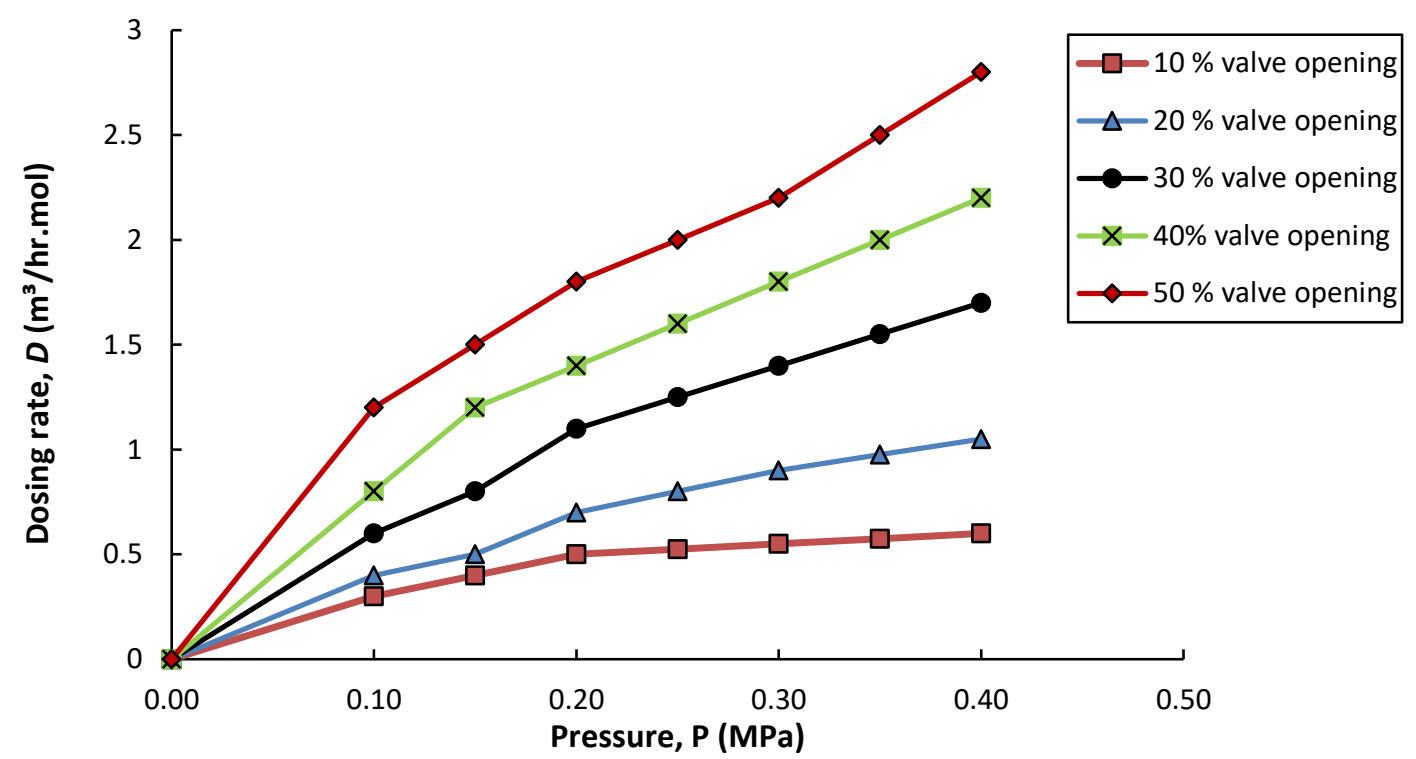

Fig. 6. Lime dosing rate measurement for 25 PPM water sample

Table 2

Airflow rate, $Q$ at different valve opening for 25 PPM

\begin{tabular}{|c|c|c|c|c|c|}
\hline \multirow{2}{*}{$\begin{array}{l}\text { Air } \\
\text { Pressure, } \\
\text { P (MPa) }\end{array}$} & \multicolumn{5}{|c|}{ Airflow rate, $\mathrm{Q}\left(\mathrm{m}^{3} / \mathrm{hr}\right)$} \\
\hline & $\begin{array}{l}10 \% \\
\text { valve } \\
\text { opening }\end{array}$ & $\begin{array}{l}20 \% \\
\text { valve } \\
\text { opening }\end{array}$ & $\begin{array}{l}30 \% \\
\text { valve } \\
\text { opening }\end{array}$ & $\begin{array}{l}40 \% \\
\text { valve } \\
\text { opening }\end{array}$ & $\begin{array}{l}50 \% \\
\text { valve } \\
\text { opening }\end{array}$ \\
\hline 0.00 & 0.00 & 0.00 & 0.00 & 0.00 & 0.00 \\
\hline 0.10 & 12.00 & 16.00 & 24.00 & 32.00 & 48.00 \\
\hline 0.15 & 16.00 & 20.00 & 32.00 & 48.00 & 60.00 \\
\hline 0.20 & 20.00 & 28.00 & 44.00 & 56.00 & 72.00 \\
\hline 0.25 & 21.00 & 32.00 & 50.00 & 64.00 & 80.00 \\
\hline 0.30 & 22.00 & 36.00 & 56.00 & 72.00 & 88.00 \\
\hline 0.35 & 23.00 & 39.00 & 62.00 & 80.00 & 100.00 \\
\hline 0.40 & 24.00 & 42.00 & 68.00 & 88.00 & 112.00 \\
\hline
\end{tabular}




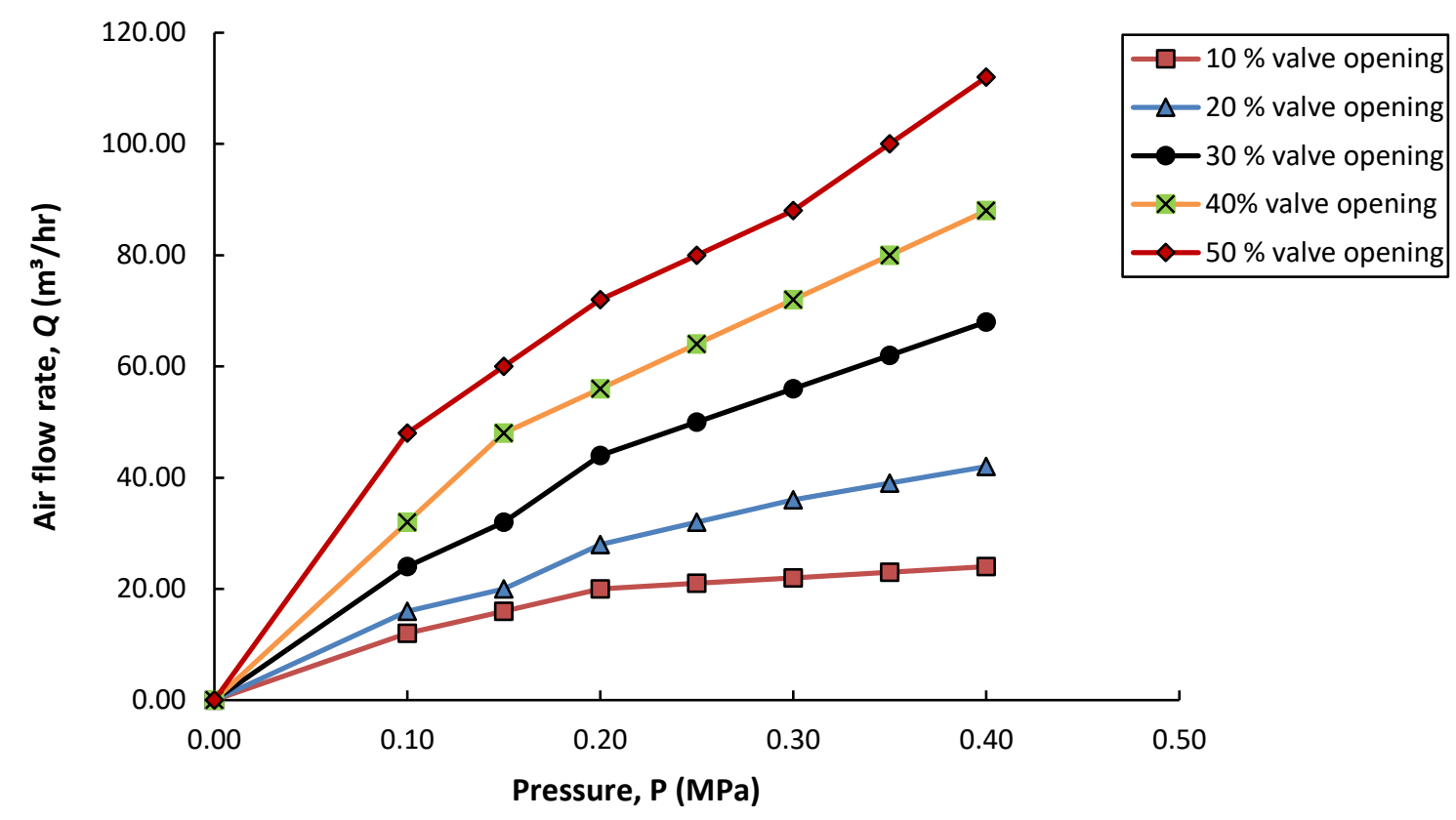

Fig. 7. Airflow rate measurement for 25 PPM water sample

\subsection{Dosing Rate Assessment on Different PPM Sampling}

This section manifests the trend of having different PPM, as it would be distinct due to the current situation of a water treatment plant [18]. At different conditions, e.g., after rain, the PPM level might go up to 50 PPM, hence changing the required lime dosing rate. On that issue, it is crucial to address the relation of the study with respect to different PPM as presented in Figure 8.

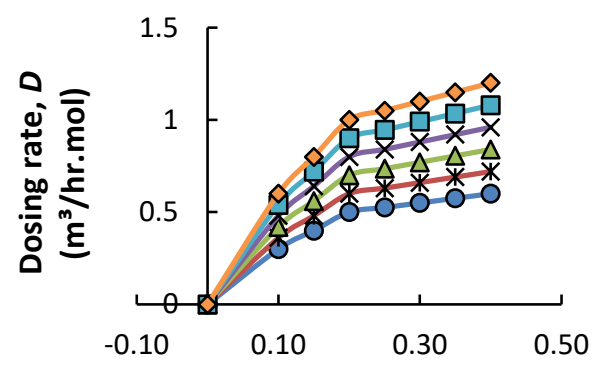

Pressure, $\mathbf{P}$ (MPa)

(a)

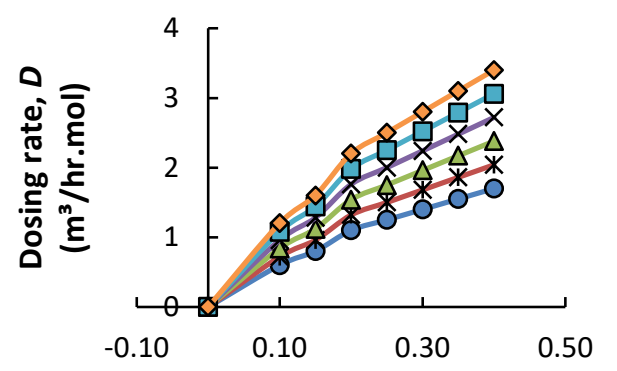

Pressure, $\mathbf{P}(\mathrm{MPa})$

(c)
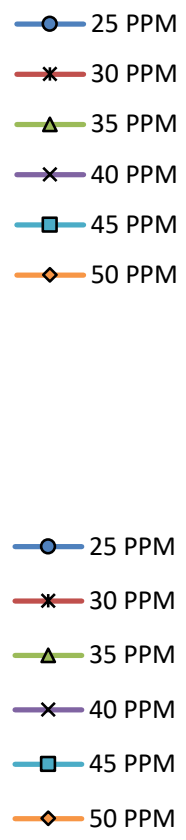
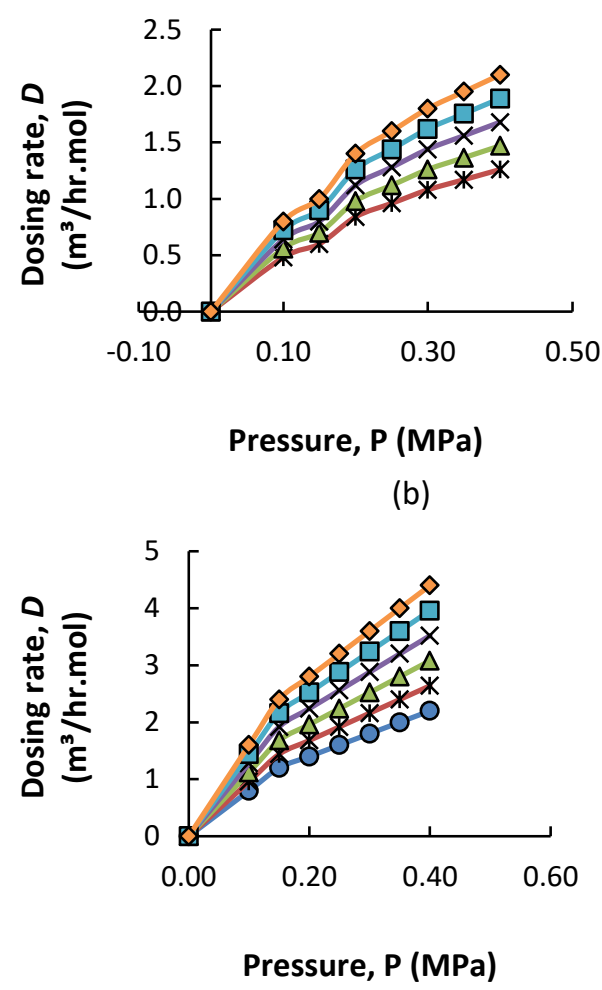

Pressure, $\mathrm{P}(\mathrm{MPa})$

(b)

(d)

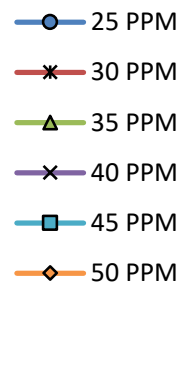

-25 PPM

* 30 PPM

$\triangle 35$ PPM

$\times 40$ PPM

$\square-45$ PPM

$\diamond 50$ PPM 


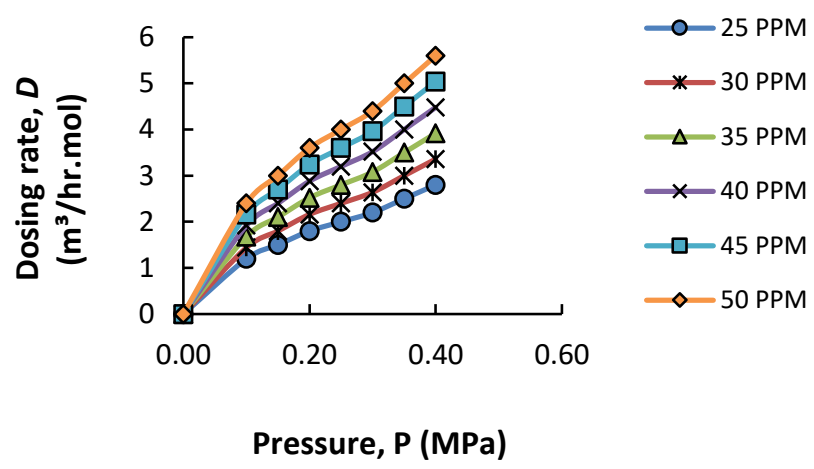

(e)

Fig. 8. Lime dosing rate estimation for respective airflow rate, $Q$ on different PPM at (a) $10 \%$ valve opening, (b) $20 \%$ valve opening, (c) $30 \%$ valve opening, (d) $40 \%$ valve opening, and (e) $50 \%$ valve opening

\section{Conclusions}

This study presents a new developed pneumatic mechanism for lime dosing system in water treatment application. The research involves such a study on different air pressure released to correlate with the lime dosing rate. On that view, a pneumatic system is adopted to obtain the required lime dosing for a certain level of airflow rate. Here, it is observed that the lime dosing rate is increased as the air pressure valve opening is increased as a decent level of pressure. In another perspective, the obtained airflow rate has been utilised to obtain the relationship behaviour of different PPM level, which is significant for a different condition in the water treatment plant.

\section{Acknowledgement}

The authors would like to express their gratitude and thanks to the Aqua Oleo Sdn Bhd to fund this research. Also, special appreciation to the IIUM River of Life (ROL) Flagship Project as this research is one of the developed projects under this program.

\section{References}

[1] Kumar, Manoj, and Avinash Puri. "A review of permissible limits of drinking water." Indian Journal of Occupational and Environmental Medicine 16, no. 1 (2012): 40. https://doi.org/10.4103/0019-5278.99696

[2] Benedict, Katharine M., Hannah Reses, Marissa Vigar, David M. Roth, Virginia A. Roberts, Mia Mattioli, Laura A. Cooley et al. "Surveillance for waterborne disease outbreaks associated with drinking water-United States, 20132014." MMWR. Morbidity and Mortality Weekly Report 66, no. $44 \quad$ (2017): 1216. https://doi.org/10.15585/mmwr.mm6644a3

[3] Liang, Jialin, Shaosong Huang, Yongkang Dai, Lei Li, and Shuiyu Sun. "Dewaterability of five sewage sludges in Guangzhou conditioned with Fenton's reagent/lime and pilot-scale experiments using ultrahigh pressure filtration system." Water Research 84 (2015): 243-254. https://doi.org/10.1016/j.watres.2015.07.041

[4] Zanin, M., H. Lambert, and C. A. du Plessis. "Lime use and functionality in sulphide mineral flotation: A review." Minerals Engineering 143 (2019): 105922. https://doi.org/10.1016/i.mineng.2019.105922

[5] Wang, Wei, Yilong Hua, Shaolin Li, Weile Yan, and Wei-xian Zhang. "Removal of Pb (II) and Zn (II) using lime and nanoscale zero-valent iron (nZVI): a comparative study." Chemical Engineering Journal 304 (2016): 79-88. https://doi.org/10.1016/i.cej.2016.06.069

[6] Sudarja, Sudarja, and Sukamta Sukamta. "Experimental Study on Flow Pattern and Void Fraction of Air-Water and 3\% Butanol Two-Phase Flow in 30 Inclined Mini Channel." Journal of Advanced Research in Experimental Fluid Mechanics and Heat Transfer 1, no. 1 (2020): 11-20.

[7] Sukamta, Sukamta, Noviyanto Noviyanto, Sudarja Sudarja, and Sri Sundari. "Characteristics of Void Fraction Using Image Processing of Two-Phase Flow of Air-Pure Water and Glycerin (40-70\%) on A Transparent Mini Pipe with 
Slope of $45^{\circ}$ to the Horizontal." Journal of Advanced Research in Experimental Fluid Mechanics and Heat Transfer 1, no. 1 (2020): 29-37.

[8] Xu, Miao, Jin-Feng Zhang, Yan-Ning Ma, Lei Yu, Xiao-Liang Liu, and Hai-Jun Feng. "Design and application of automatic lime dosing system in metal pickling wastewater treatment." Industrial Water \& Wastewater 6 (2011).

[9] Zhang, Ning, MinGuan Yang, Bo Gao, Zhong Li, and Dan Ni. "Experimental investigation on unsteady pressure pulsation in a centrifugal pump with special slope volute." Journal of Fluids Engineering 137, no. 6 (2015). https://doi.org/10.1115/1.4029574

[10] Chang, Chia-Jung, Chin-Pao Huang, Chia-Yang Chen, and Gen-Shuh Wang. "Assessing the potential effect of extreme weather on water quality and disinfection by-product formation using laboratory simulation." Water Research 170 (2020): 115296. https://doi.org/10.1016/i.watres.2019.115296

[11] Pasha, Amjad Ali, Meshal Nuwaym Al-Harbi, Surfarazhussain S. Halkarni, Nazrul Islam, D. Siva Krishna Reddy, S. Nadaraja Pillai, and Ufaith Qadiri. "CFD study of Convective Heat Transfer of Water Flow Through Micro-Pipe with Mixed Constant Wall Temperature and Heat Flux Wall Boundary Conditions." CFD Letters 13, no. 7 (2021): 13-26. https://doi.org/10.37934/cfdl.13.7.1326

[12] Mintenig, S. M., M. G. J. Löder, Sebastian Primpke, and Gunnar Gerdts. "Low numbers of microplastics detected in drinking water from ground water sources." Science of the Total Environment 648 (2019): 631-635. https://doi.org/10.1016/i.scitotenv.2018.08.178

[13] Ke, lou-Sheng, Mykhaylo Myahkostupov, Felix N. Castellano, and François P. Gabbaï. "Stibonium ions for the fluorescence turn-on sensing of F-in drinking water at parts per million concentrations." Journal of the American Chemical Society 134, no. 37 (2012): 15309-15311. https://doi.org/10.1021/ja308194w

[14] Nariyan, E., N. Aravindakshan, Q. J. Yu, and Q. Li. "Removal of iodides and bromides at parts per million concentrations using a novel bismuth composite material." Materials Today Sustainability 10 (2020): 100054. https://doi.org/10.1016/i.mtsust.2020.100054

[15] Chen, Shengzhi, Chongho Youn, Toshiharu Kagawa, and Maolin Cai. "Transmission and consumption of air power in pneumatic system." Energy and Power Engineering 6, no. 13 (2014): 487-495. https://doi.org/10.4236/epe.2014.613042

[16] Shi, Yan, Maolin Cai, Weiqing Xu, and Yixuan Wang. "Methods to Evaluate and Measure Power of Pneumatic System and Their Applications." Chinese Journal of Mechanical Engineering 32, no. 1 (2019): 42-42. https://doi.org/10.1186/s10033-019-0354-6

[17] Bonton, Alexandre, Christian Bouchard, Benoit Barbeau, and Stéphane Jedrzejak. "Comparative life cycle assessment of water treatment plants." Desalination $284 \quad$ (2012): 42-54. https://doi.org/10.1016/i.desal.2011.08.035

[18] Lin, Shun Dar. Water and wastewater calculations manual. McGraw-Hill Education, 2014. 